\title{
Non-volatile bistability effect based on electrically controlled phase transition in scaled magnetic semiconductor nanostructures
}

\author{
Y. G. Semenov and K. W. Kim \\ Department of Electrical Computer Engineering, \\ North Carolina State University, Raleigh, NC 27695-7911
}

\begin{abstract}
We explore the bistability effect in a dimensionally scaled semiconductor nanostruncture consisting of a diluted magnetic semiconductor quantum dot (QD) and a reservoir of itinerant holes separated by a barrier. The bistability stems from the magnetic phase transition in the QD mediated by the changes in the hole population. Our calculation shows that when properly designed, the thermodynamic equilibrium of the scaled structure can be achieved at two different configurations; i.e., the one with the QD in a ferromagnetic state with a sufficient number of holes and the other with the depopulated QD in a paramagnetic state. Subsequently, the parameter window suitable for this bistability formation is discussed along with the the conditions for the maximum robustness/non-volatility. To examine the issue of scaling, an estimation of the bistabiity lifetime is made by considering the thermal fluctuation in the QD hole population via the spontaneous transitions. A numerical evaluation is carried out for a typical carrier-mediated magnetic semiconductor (e.g., GaMnAs) as well as for a hypothetical case of high Curie temperature for potential room temperature operation.
\end{abstract}

PACS numbers: 72.20.Ht,85.60.Dw,42.65.Pc,78.66.-w 
The magnetism in semiconductors is the basis for the emerging field of spin-polarized electronics, or spintronics. $\stackrel{1}{=}$ Substantial progress has been made during the past few years, particularly in the materials development. The advantages of the semiconductor-based systems over the metallic counterparts include the controllability of the ferromagnetism (via the bias $^{2}$ and/or doping) and the potential compatibility with the modern Si-based processing technology.

Recently, a theoretical study further explored the opportunities offered by the electrically controlled magnetism. The calculation found that a properly designed structure consisting of magnetic and non-magnetic semiconductor quantum wells (QWs) can exhibit the bistability with respect to the paramagnetic-ferromagnetic (PM-FM) phase transition when the process is controlled by the itinerant carriers (holes) $\stackrel{\underline{3}}{\underline{3}}$ This bistability effect in the two-dimensional system was predicted to persist even at temperatures nearly as high as the critical temperature $T_{c}$ of the PM-FM phase transition. Subsequently, a non-volatile memory application was suggested citing the successful growth of transition metal doped semiconductors that are FM at or above room temperature (e.g., the nitrides,,$\underline{4}$ Ge,,$\underline{5}$ as well as some II-VI's ${ }^{6.7}$ ).

For practical realization of the proposed device application, it is highly desirable to reduce the size of the magnetic layer (i.e., the active part of the memory) without the loss of high temperature operability and non-volatility. Consequently, a magnetic semiconductor quantum dot (QD) that can exchange itinerant holes with a reservoir provides an interesting opportunity. However, unlike the QW case examined earlier, $\stackrel{3}{=}$ the thermal fluctuation resulting from spontaneous hopping between the two possible stable states cannot be neglected in the scaled structure due to the limited number of carriers populating the QD. Hence, it is the purpose of this paper to investigate theoretically the effect of the reduced dimensionality and explore the potential bistability conditions based on the electrically controlled magnetic phase transition in the magnetic semiconductor nanostructures.

The specific structure under consideration consists of a single diluted magnetic semiconductor (DMS) QD separated from a large reservoir of itinerant holes, which controls the chemical potential $\mu_{0}$ of the system. For simplicity, we assume $\mu_{0} \gg k_{B} T$ and ignore the possible temperature dependence of $\mu_{0}$. A non-magnetic QW filled with itinerant holes (for example, through the modulation doping, etc.) can be used as the desired reservoir (see Fig. 1).

To accurately describe this system, one needs to know the energy structure of the QD 
that is a function of multiple parameters. Particularly, the magnetic interactions must be taken into account that leads to the PM-FM phase transition in the DMS QD. Note that the analysis of different magnetic phase transition mechanisms is beyond the scope of the present study (see, for details, Ref. 8). Instead, we assume that the main magnetic properties [such as the critical temperature $T_{c}$ and its dependence on the hole concentration, and the magnetization dependence on temperature $M=M(T)]$ can be obtained from the measurements of the relevant DMS. This allows the use of a semi-phenomenological approach to calculate the free energy of the system.

In the present model, we approximate the free energy of the QD as the sum of two terms, the magnetic $\left(F_{M}\right)$ and non-magnetic $\left(F_{N}\right)$ contributions. If the DMS QD is not far from the PM-FM transition, the Landau expansion over the magnetization $M$ can be applied for $F_{M}$ :

$$
F_{M}=-a\left(T_{c}-T\right) M^{2}+b M^{4}
$$

The parameters $a, b$ and $T_{c}$ are the functions of hole population $j$ in the QD; particularly, the dependence $T_{c}=T_{c}(j)$ plays a crucial role in the magnetic instability $\frac{9}{-}$ In addition, $a$ and $b$ can be expressed in terms of the fundamental properties of the magnet: The Curie-Weiss law for magnetic susceptibility $\chi=C_{0} /\left(T-T_{c}\right)$ at $T>T_{c}$ defines $a=1 / 4 C_{0}$, while spontaneous magnetization $M_{s}=M_{0} \sqrt{1-T / T_{c}}$ at $T<T_{c}$ provides $b=a T_{c} / 2 M_{0}^{2}$. Since Eq. (10) is assumed to fully describe the magnetization of the DMS QD, the thermodynamically stable state corresponds to the specific magnitude of $M$ that gives rise to the free energy minimum

$$
F_{M}=-T_{c} \frac{M_{0}^{2}}{C_{0}}\left(1-\frac{T}{T_{c}}\right)^{2} \theta\left(1-\frac{T}{T_{c}}\right),
$$

where $\theta(x)$ is the Heaviside step function. It is important to note that the localized spin $S$ of the magnetic ions provides the main contribution to the DMS magnetization, whereas that of the itinerant carriers add a minor role. The parameters $M_{0}$ and $C_{0}$ can be easily estimated for $N_{m}$ localized spin moments leading to the estimation $M_{0}^{2} / C_{0}=3 S N_{m} / 8(S+1)$, which is independent of carriers. Thus, the only dependence of $F_{M}$ on the hole population $j$ comes from the term $T_{c}=T_{c}(j)$ in our approximation.

To obtain a numerical value for $j$, one must also incorporate the non-magnetic part $F_{N}$ of the free energy for $j$ particles located in the QD. Unfortunately, the calculation of $F_{N}$ requires the approaches very specific to each individual case as it depends on the details such as the material composition, size and shape of the QD, presence of dopants and external 
fields, etc. Consequently, this problem cannot be solved in a general manner. To proceed further, we treat the QD as a scaled QW with a finite lateral size (and thickness); this is analogous to a nanodot embedded in a barrier.

The potential profile of the sample structure along the growth $(z)$ direction is schematically illustrated in Fig. 1 from the hole representation, as is the case throughout the paper. It is convenient to split $F_{N}$ into two parts, $F_{N}=E_{j}+F_{1}(T, j)$. The first term,

$$
E_{j}=j U+\frac{1}{2} j(j-1) C
$$

accounts for the energy acquired by $j$ particles due to their localization in the QD with the ground state energy $U$ as well as their Coulomb repulsion energy $\left(C=e^{2} / \epsilon \sqrt{A_{0}}\right.$, where $e$ is the electron charge, $\epsilon$ the dielectric constant, and $A_{0}$ the lateral cross-section of the QD) $!^{10}$ Then, the remaining part,

$$
F_{1}(T, j)=\Omega\left(T, \mu_{1}\right)+j \mu_{1}(j)
$$

is similar to the free electron gas contribution with the thermodynamic potential ${ }^{11}$

$$
\Omega\left(T, \mu_{1}\right)=-k_{B} T \sum_{n} \ln \left[1+e^{\left(\mu_{1}-\varepsilon_{n}\right) / k_{B} T}\right]
$$

$\varepsilon_{n}$ and $\mu_{1}$ represent the energy spectrum (with the quantum number $n$ ) and the chemical potential of the QD when the influence of the magnetic interaction is excluded (i.e., the non-magnetic version of the QD). Hereinafter, we consider the lateral dimension $A_{0}$ of the QD to be relatively sizable so that the energy gaps in the discrete energy spectra are smaller than $k_{B} T$. Then, the sum in Eq. (5) can be approximated by an integral with the density of states $m A_{0} / \pi \hbar^{2}$. From the relation $j=-\partial \Omega\left(T, \mu_{1}\right) / \partial \mu_{1}, \mu_{1}(j)$ is found to be

$$
\mu_{1}(j)=k_{B} T \ln \left(e^{\xi j}-1\right)
$$

where $\xi=\pi \hbar^{2} / m A_{0} k_{B} T$ and $m$ is the in-plane hole effective mass. Subsequently, the thermodynamic potential [Eq. (5)] can be expressed in the form

$$
\Omega\left[T, \mu_{1}(j)\right]=-\frac{k_{B} T}{\xi} \int_{0}^{\infty} \ln \left[1+\left(e^{\xi j}-1\right) e^{-x}\right] d x .
$$

Equations (3) and (44) along with $\mu_{1}(j)$ and $\Omega\left(T, \mu_{1}\right)$ from Eqs. (6) and (17) determine the non-magnetic part of the free energy, while the total free energy of the DMS QD is the sum $F=F_{M}+F_{N}$. 
Now we take into account that the QD is in contact with a large reservoir providing two-way exchange of carriers through the potential barrier (see Fig. 1). This leads to the establishment of a unified chemical potential that coincides with $\mu_{0}$ of the reservoir. Thus, the equation that determines the population of the $\mathrm{QD}$ takes the form

$$
\mu_{Q D}(j)=\mu_{0}
$$

Note that $\mu_{Q D}(j) \neq \mu_{1}(j)$ since both the non-magnetic and magnetic interactions are considered for $\mu_{Q D}(j)$. Since the chemical potential $\mu_{Q D}(j)$ of the QD can be expressed as $\mu_{Q D}(j)=d F / d j$ in general, the stable solutions of Eq. (8) must correspond to the local minima of $F=F(j)$ or equivalently $d \mu_{Q D}(j) / d j>0$.

Finally, the desired solutions require a specific expression for the dependence $T_{c}=T_{c}(j)$ in Eq. (21). Following the experimental data of Ref. 12, we adopt a semi-phenomenological description

$$
T_{c}=T_{c}^{0}\left(1-e^{-\alpha j \xi t}\right)
$$

where $T_{c}^{0}$ is the asymptotic value of the critical temperature (at a sufficiently high hole concentration), $t=T / T_{c}^{0}$, and $\alpha(=1)$ is the fitting parameter that adjusts Eq. (9) to the experiments. Subsequently, the QD population $j$ can be obtained from Eqs. (2), (3), (41), and (8) as

$$
\begin{aligned}
\mu_{0}-U= & (j-1 / 2) C+k_{B} T \ln \left(e^{\xi j}-1\right)- \\
& k_{B} T \xi \frac{3 S N_{m}}{8(S+1)} e^{-\alpha \xi t j}\left[1-\left(\frac{T}{T_{c}}\right)^{2}\right] \theta\left(1-\frac{T}{T_{c}}\right) .
\end{aligned}
$$

For a numerical evaluation, let us assume the following set of parameters "typical" for a carrier mediated DMS (e.g., $\left.\mathrm{Ga}_{0.95} \mathrm{Mn}_{0.05} \mathrm{As}\right): m=0.13 m_{0}\left(m_{0}\right.$ is the free electron mass), $\epsilon=12.9, S=5 / 2, N_{m}=1.3 \times 10^{21} \mathrm{~cm}^{-3} \times(\mathrm{QD}$ volume $)$, and $T_{c}^{0}=110 \mathrm{~K}$. Figure 2 depicts the dependence $\left[\mu_{Q D}(j)-U\right] / k_{B} T_{c}^{0}$ vs. $j$. Clearly, the results indicate that only one solution for $j$ exists at sufficiently low or high energies $U$ in reference to $\mu_{0}$ (e.g., dashed line 1 or 3) corresponding to the only stable QD population at a given $U$. However, the moderate values of $U$ can support multiple roots. In the case of dashed line 2, two of them (with the smallest and largest $j)$ are stable considering the positive derivative $\left(d \mu_{Q D} / d j>0\right)$, while the intermediate solution is not $\left(d \mu_{Q D} / d j<0\right)$. Note also that the stable solutions with the larger (smaller) $j$ are realized in the FM (PM) phase of the DMS QD. Hence, this 
demonstrates a bistable state for a properly designed QD in terms of the hole population or the magnetic phase. Figure 3 provides the ranges of $U$ (assuming a fixed $\mu_{0}$ ) and $T$ where the bistability can be expected in the system under consideration. Clearly, the calculation results indicates a large window in the system parameter space where the bistability is possible. The maximum operating temperature may be nearly as high as $T_{c}^{0}$.

To achieve a bistable state robust against the thermal fluctuations (i.e., non-volatile) for the possible memory application, it is necessary to select a condition that provides the maximal separation $\Delta F=\min \left\{F_{\max }-F_{P \min }, F_{\max }-F_{F \min }\right\}$ between the local maximum $F_{\max }$ and each of the local minima $F_{P \text { min }}$ and $F_{F \text { min }}$ (for the PM and FM phase of the DMS $\mathrm{QD}$, respectively) of the free energy $F(j)$. At a given temperature, one can find the $\mathrm{QD}$ potential alignment that results in the maximal $\Delta F$. Figure 4 illustrates the behavior of $F(j)$ at three different shifts $\Delta \mu=\mu_{0}-U$ : curve 1 (3) represents the case of the monostable state in the FM (PM) state, whereas curve 2 exhibits two local minima in the FM and PM states, respectively, separated by the maximal $\Delta F$. The temperature dependence of the maximal $\Delta F$ and the associated optimal potential shift $\Delta \mu$ is plotted in Fig. 5 along with the mean values of the particle numbers $j_{P}, j_{m}$ and $j_{F}$ corresponding to $F_{P \min }, F_{\max }$ and $F_{F \text { min }}$. Our analysis shows that the "thermodynamic barrier" $\Delta F$ decreases drastically as $T$ approaches $T_{c}^{0}$ [curve 2 in Fig. 5(a)]. Obviously, the system based on the PM-FM transition becomes much less stable near the critical temperature.

Note that the mean value $j$ is reached through the balance of the particle flux to and from the QD. Each of these incidents transfers one particle via the thermally activated processes with a characteristic time $\tau_{0}$, which depends on the temperature, height and width of the energy barrier separating the $\mathrm{QD}$ and reservoir, etc. The lifetime $T_{l t}$ is defined as the time it takes to develop a sufficiently large fluctuation to induce a transition from the state initially at $F_{F \text { min }}=F\left(j_{F}\right)$ to that at $F_{P \text { min }}=F\left(j_{P}\right)$. Apparently if the system reaches the state at the local maximum $F_{\max }=F\left(j_{m}\right)$, further evolution can result in either the PM (with $j=j_{P}$ ) or the FM (with $j=j_{F}$ ) QD state with an approximately equal probability; hence, $T_{l t}$ can be estimated as the reciprocal probability for the process $F_{F \min } \rightarrow F_{\max }$ or $j_{F} \rightarrow j_{m}$.

The formation of fluctuation $\Delta j=j_{F}-j_{m}$ can be considered based on the sequential process of hole withdrawal from the QD. For the first hole transfer out of the QD, the characteristic time of this process is $\tau_{0}$ as defined above. Due to the finiteness of the QD hole population, this reduces the chemical potential by $\Delta \mu(1)=\mu_{Q D}\left(j_{F}\right)-\mu_{Q D}\left(j_{F}-1\right)$. 
Consequently, the new time constant becomes $\tau_{0} \exp \left[\Delta \mu(1) / k_{B} T\right]$ when the next hole escapes from the $\mathrm{QD}$, provided no particles are injected into the $\mathrm{QD}$ from the reservoir. Hence, the mean time necessary for the $\Delta j$ fluctuation through the sequential withdrawal can be estimated as

$$
T_{w}(\Delta j)=\tau_{0} \sum_{j=0}^{j_{m}-1} \exp \left[\Delta \mu(j) / k_{B} T\right] .
$$

On the other hand, the probability of no hole injection from the reservoir during this time span $T_{w}$ is $P_{w}=\exp \left[-T_{w}(\Delta j) / \tau_{0}\right]$. The frequency of appearance for such a rare occasion is $P_{w} / \tau_{0}$ and the desired lifetime is

$$
T_{l t}=\tau_{0} \exp \left[T_{w}(\Delta j) / \tau_{0}\right]
$$

A similar expression is shown to apply to the fluctuations $F_{P \min } \rightarrow F_{\max }$ or $j_{P} \rightarrow j_{m}$.

One can see that the estimated lifetime (or the bit retention time) depends crucially on the operating temperature and the QD sizes, which determine the number of terms $j_{m}$ in the sum of Eq. (11). Figure 6 analyzes the results for the GaMnAs QD with the dimension of $25 \times 25 \times 5 \mathrm{~nm}^{3}$ and $15 \times 15 \times 5 \mathrm{~nm}^{3}$ assuming $T_{c}^{0}=110 \mathrm{~K}$. As expected, the bistable state becomes less stable (i.e., shorter $T_{l t}$ ) as the QD size shrinks. The thermal fluctuation clearly has a bigger impact in this case due to the finite number of holes in the QD. For the two structures considered (or those of similar sizes), a practically non-volatile condition (i.e., sufficiently long bit retention) may be achieved when operating below approx. $75 \mathrm{~K}$. $\tau_{0}=1 \mathrm{~ns}$ is used for the calculation.

To examine the feasibility of room temperature application, it is desirable to extend our consideration of magnetic semiconductors to those emerging ones with potentially much higher critical temperatures. Note for instance two recent reports of the DMS with $T_{c} \geq$ $300 \mathrm{~K} \cdot 5.7$ Since the search for an ideal DMS is only at the beginning stage, we assume a hypothetical material with the characteristics similar to GaMnAs except $T_{c}^{0}$ in Eq. (9), which is treated as a variable in a wide temperature range. Figure 7 depicts the estimated lifetime as a function of $T_{c}^{0}$, while $T$ is fixed at $300 \mathrm{~K}$. For a sufficiently long $T_{l t}$ in this case, the desired material needs $T_{c}^{0}$ of approx. $550 \mathrm{~K}$ or higher.

In summary, we investigate theoretically the effect of the reduced dimensionality and explore the potential bistability conditions based on the electrically controlled magnetic phase transition in the magnetic semiconductor nanostructures. The analysis is based on 
a semi-phenomenological model that assumes the common magnetic behavior and a simple hole energy spectrum in a DMS QD. When properly designed, the calculation predicts the possibility of controlled switching between the stable PM and FM states in the QD. The parameter window suitable for the bistability formation is discussed along with the conditions for the maximum robustness/non-volatility. An estimation of the bistability lifetime limited by the thermal fluctuation provides a guideline for its potential application as a room temperature low-power, high-density memory element.

This work was supported in part by the Defense Advanced Research Projects Agency and the SRC/MARCO Center on FENA. 
1 Spin Electronics, edited by D. Awschalom (Kluwer, Dordrecht, 2004).

2 H. Ohno, D. Chiba, F. Matsukura, T. Omiya, E. Abe, T. Dietl, Y. Ohno, and K. Ohtani, Nature 408, 944 (2000); D. Chiba, M. Yamanouch, F. Matsukura, and H. Ohno, Science 301, 943 (2003).

3 Y. G. Semenov, H. Enaya, and K. W. Kim, Appl. Phys. Lett. 86, 073107 (2005).

4 M. L. Reed, N. A. El-Masry, H. H. Stadelmaier, M. K. Ritums, M. J. Reed, C. A. Parker, J. C. Roberts, and S. M. Bedair, Appl. Phys. Lett. 79, 3473 (2001).

5 F. Tsui, L. He, L. Ma, A. Tkachuk, Y. S. Chu, K. Nakajima, and T. Chikyow, Phys. Rev. Lett. 91, 177203 (2003).

6 H. Saito, V. Zayets, S. Yamagata, and K. Ando, Phys. Rev. Lett. 90, 207202 (2003).

7 N. S. Norberg, K. R. Kittilstved, J. E. Amonette, R. K. Kukkadapu, D. A. Schwartz, and D. R. Gamelin, J. Am. Chem. Soc. 126, 9387 (2004).

8 E. A. Pashitskii and S. M. Ryabchenko, Fiz. Tverd. Tela, 21, 545 (1979) [Sov. Phys. Solid State, 21, 322 (1979)]; T. Dietl, H. Ohno, and F. Matsukura, Phys. Rev. B 63, 195205 (2001); J. Fernández-Rossier and L. J. Sham, Phys. Rev. B 64, 235323 (2001); V. I. Litvinov and V. K. Dugaev, Phys. Rev. Lett. 86, 5593 (2001); A. Kaminski and S. Das Sarma, Phys. Rev. Lett. 88, 247202 (2002); A. C. Durst, R. N. Bhatt, and P. A. Wolff, Phys. Rev. B 65, 235205 (2002); Y. G. Semenov and V. A. Stephanovich, Phys. Rev. B 66, 075202 (2002); P. M. Krstajić, F. M. Peeters, V. A. Ivanov, V. Fleurov, and K. Kikoin, Phys. Rev. B 70195215 (2004).

9 F. Guinea, G. Gomez-Santos, and D. P. Arovas, Phys. Rev. B 62, 391 (2000).

10 Refining the Coulomb term results in small corretions to the numerical results.

11 Here we go from the canonical to the grand canonical Gibbs ensemble treating $j$ as the mean value $\langle j\rangle$ over the ensemble.

12 H. Boukari, P. Kossacki, M. Bertolini, D. Ferrand, J. Cibert, S. Tatarenko, A. Wasiela, J. A. Gaj, and T. Dietl, Phys. Rev. Lett. 88, 207204 (2002). 


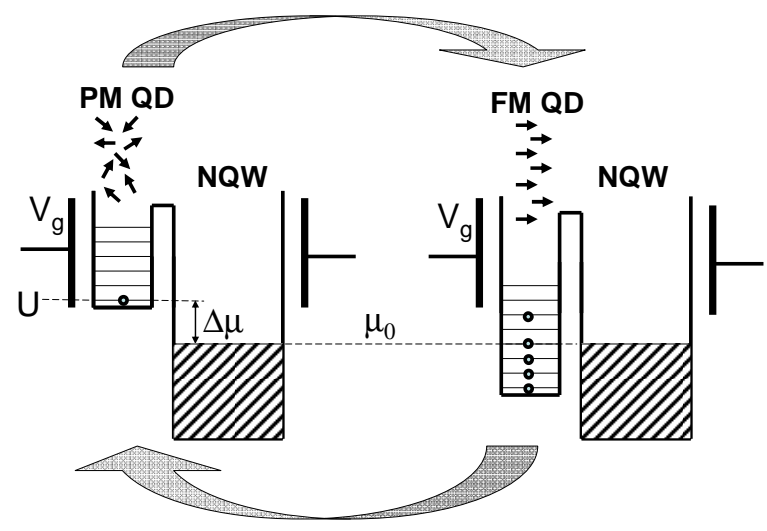

FIG. 1: Schematic diagram of the structure containing a DMS QD (in two different phases) and a nonmagnetic quantum well (NQW) reservoir separated by a barrier. The energy orientation is provided from the hole point of view in the valence band. Left: The PM phase corresponds to disordered magnetic ion spins (small arrows) and the lack of the magnetic contribution to the hole energy. This is achieved when the holes (small circles) only weakly populate the QD with a discrete energy spectrum and, thus, cannot change its magnetic state. The ground state $U$ of the QD is relatively high in reference to the chemical potential $\mu_{0}$ of the hole reservoir. Right: Another thermodynamically stable state (at the same external conditions) is possible when the magnetic ions are ferromagnetically ordered. Magnetic interactions can decrease the hole potential that the ground state of the DMS QD is now sufficiently below $\mu_{0}$; the equilibrium hole population is high enough to stabilize the FM phase. Switching between the PM and FM states can be achieved by applying a gate bias $V_{g}$ that populates or depopulates the DMS QD. 


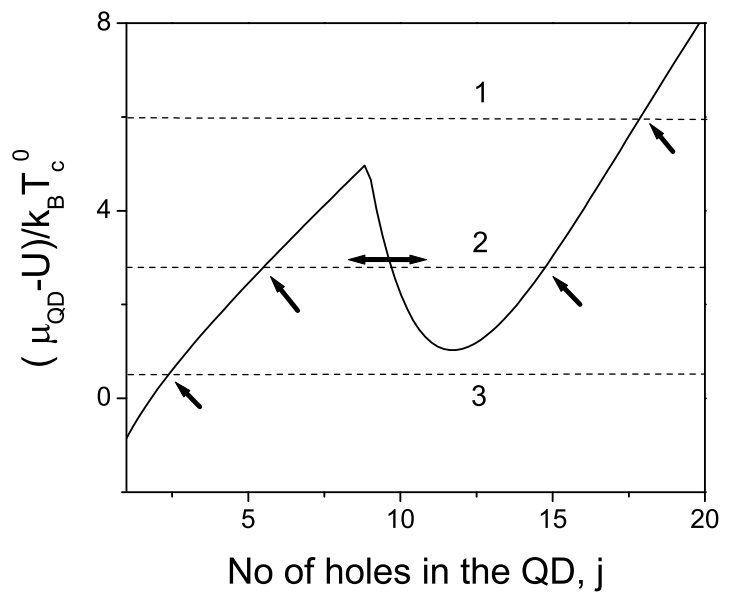

FIG. 2: Chemical potential of the DMS QD with the thickness of $5 \mathrm{~nm}$ and the cross-section of $25 \times 25 \mathrm{~nm}^{2}$ as a function of the QD hole population [Eq. [10)]. The parameters of $\mathrm{Ga}_{0.95} \mathrm{Mn}_{0.05} \mathrm{As}$ are assumed with $T=77 \mathrm{~K}$ as discussed in the text. The solutions of Eq. (10) can be found as intersections of the solid curve with the horizontal line corresponding to a certain value of $\Delta \mu$ $\left(=\mu_{0}-U\right)$. Two cases $\Delta \mu / T_{c}^{0}=6$ and 0.5 (dashed lines 1 and 3) provide monostable FM and PM states, while dashed line $2\left(\Delta \mu / T_{c}^{0}=2.7\right)$ depicts the bistable state. Stable solutions are indicated by single-head arrows and the unstable one by the horizontal double-head arrow. 


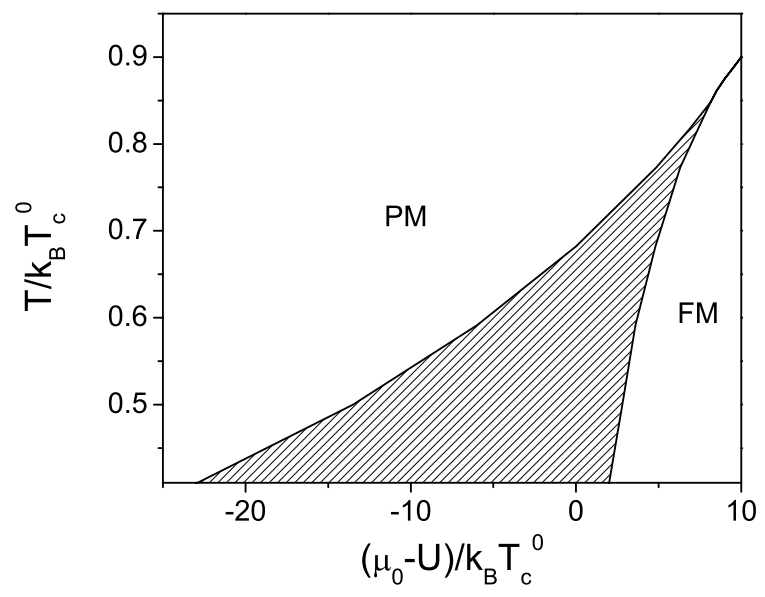

FIG. 3: Phase diagram of the parameter space indicating the potential bistability region (the shaded area). The PM and FM denote the monostable areas corresponding to the PM and FM QD states, respectively. The same parameters as in Fig. 2 are assumed $\left(T_{c}^{0}=110 \mathrm{~K}\right)$. 


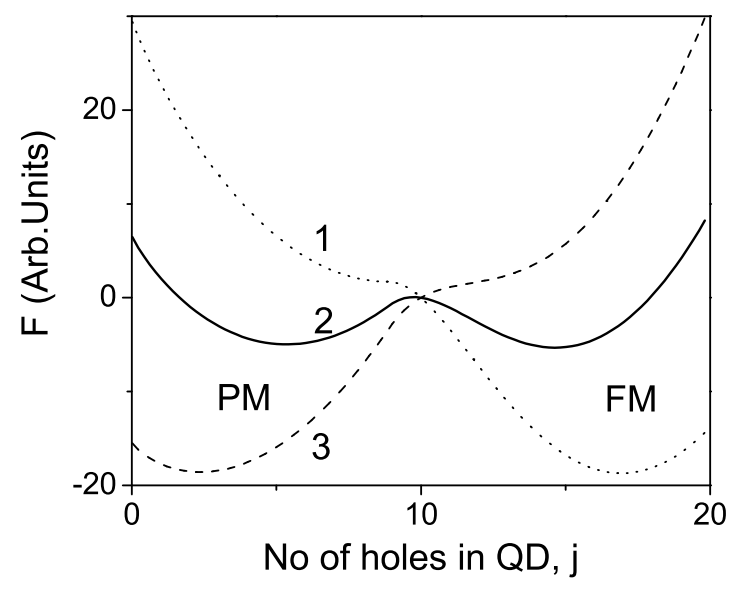

FIG. 4: Free energy of the QD calculated as a function of hole population for three different values of $\Delta \mu\left(=\mu_{0}-U\right): \Delta \mu / T_{c}^{0}=6$ (curve 1, dotted line); 2.7 (curve 2, solid line); 0.5 (curve 3, dashed line). The single minima of curves 1 (FM phase) and 3 (PM phase) correspond to the vicinities of the right and left boundaries of the bistable area in Fig. 3; curve 2 represent the bistable case with the optimal free energy barrier height separating two local minima. The same parameters as in Fig. 2 are assumed $\left(T=77 \mathrm{~K}, T_{c}^{0}=110 \mathrm{~K}\right)$. 


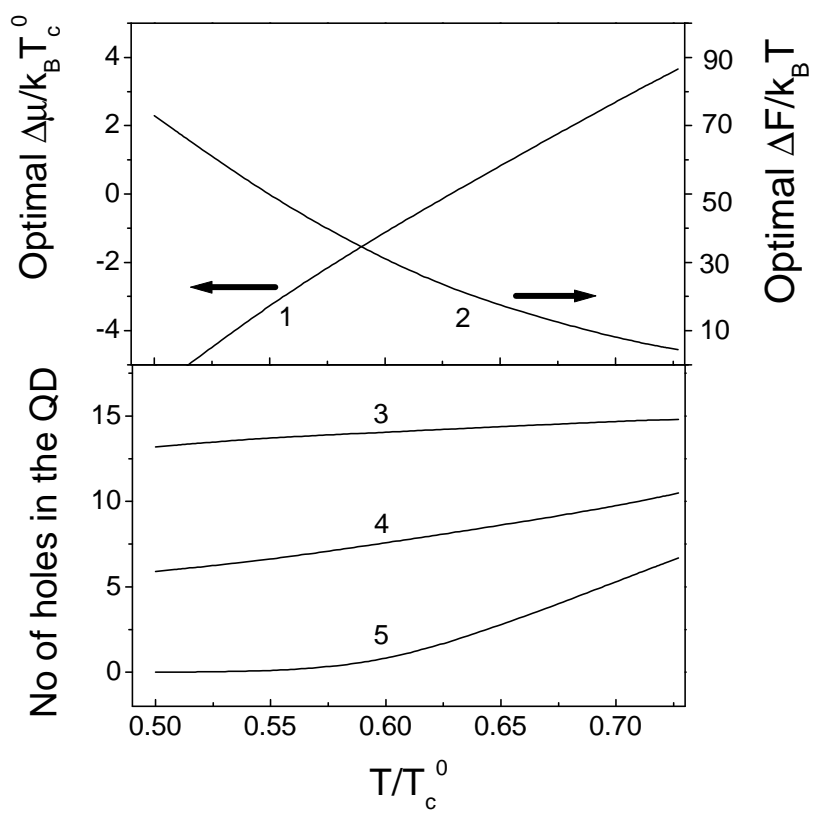

FIG. 5: Maximal free energy barrier height (in units of $k_{B} T$ ) between the local maximum and the closest local minimum in the bistable case (curve 2) and the corresponding optimal chemical potential (curve 1) as a functions of temperature. Curves $3-5$ in the bottom pane shows the hole population at the FM minimum (curve 3), the PM minimum (curve 5), and the local maximum (curve 4 ) of $F(j)$ for the bistable case shown in the top pane at the corresponding temperature. The same parameters as in Fig. 2 are assumed $\left(T_{c}^{0}=110 \mathrm{~K}\right)$ 


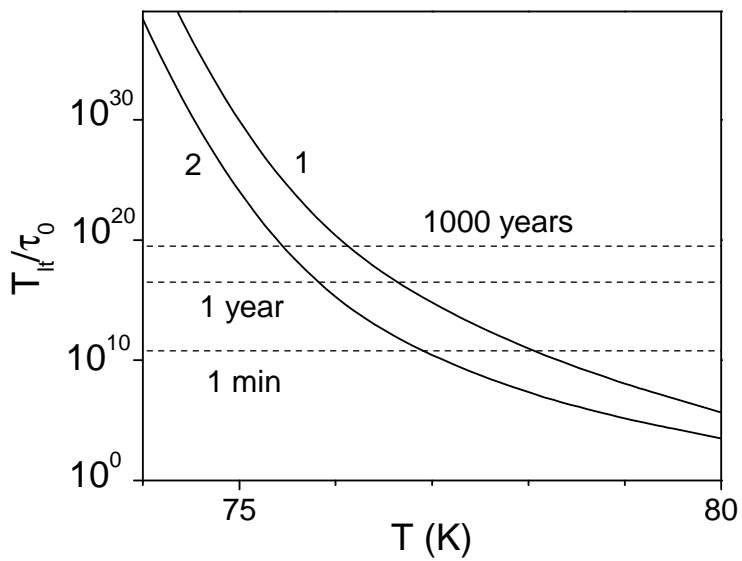

FIG. 6: Bistability lifetime vs. temperature at $T_{c}^{0}=110 \mathrm{~K}$. Two different QD dimensions are considered: (1) $25 \times 25 \times 5 \mathrm{~nm}^{3}$ and (2) $15 \times 15 \times 5 \mathrm{~nm}^{3}$. The mean time $\tau_{0}$ of particle exchange between the QD and reservoir via thermal processes is assumed to be $1 \mathrm{~ns}$. Other parameters are the same as in Fig. 2. 


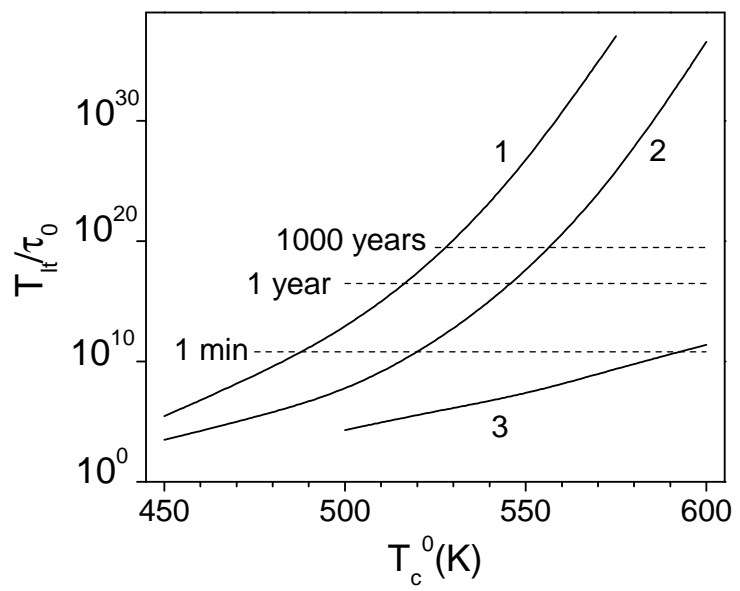

FIG. 7: Bistability lifetime vs. temperature for a hypothetical DMS material with the characteristics similar to GaMnAs except $T_{c}^{0}$ in Eq. (9), which is treated as a variable in a wide temperature range. $T$ is fixed at $300 \mathrm{~K}$ and three different QD sizes are considered: (1) $25 \times 25 \times 5 \mathrm{~nm}^{3}$; (2) $15 \times 15 \times 5 \mathrm{~nm}^{3}$; and (3) $25 \times 25 \times 3 \mathrm{~nm}^{3}$. The mean time $\tau_{0}$ of particle exchange between the QD and reservoir via thermal processes is assumed to be 1 ns as in Fig. 6 